\title{
The Role of EVTM in the Austere Environment
}

\author{
Mohammed Al-Musawi MBChB, MSc, FIBMS-CTV, FRCS “Glasg” \\ Research Faculty, Department of surgery/Division of Cardiothoracic Surgery, School of Medicine, University of Colorado, USA
}

In November 2019 we, as a group of Iraqi cardiovascular surgeons, were invited to give a lecture in the Pan-American Endovascular Resuscitation and Trauma Management (EVTM) [1] symposium in Denver, Colorado to share our experience in management of mass causalities in the austere environment. The main topic we tried to highlight was how we approached bleeding control and resuscitation with scarcity of resources while dealing with large volumes of trauma cases. Before attending this symposium we did not know what EVTM was or what it stood for. That EVTM was a two-day symposium and our lecture was last; therefore, we had the ability to learn more about its principles and goals. We were surprised to find that we use the same principles in our daily practices in Iraq without calling it EVTM. The main difference is our scarcity of resources and how we modify less sophisticated catheters or balloons to help control bleeding or to resuscitate patients during their transfer. Of course, the main principles are the same regarding early prehospital trauma care, which is one of the most important measures to improve outcome in trauma surgery [2]. This includes securing airways, packing wounds, using tourniquets for bleeding limbs, getting intravenous access with fluid replacement, stabilizing fractures and a quick transfer to the nearest medical facility [3]. Unfortunately, in our daily practice in Iraq we don't get all of these steps done - sometimes some of them are done but most of the time none of them. One example of improvisation is the use of simple occlusion catheters or the Fogarty catheter either during patient transfer or peroperatively to stop exsanguination and help resuscitation. We notice in our practice that the impact of poor prehospital care significantly

\section{Corresponding author:}

Mohammed Al-Musawi MBChB, MSc, FIBMS-CTV, FRCS "Glasg",

Research Faculty, Department of surgery/Division of Cardiothoracic Surgery, School of Medicine, University of Colorado, USA.

Email: mohammed.al-musawi@cuanschutz.edu

(C) 2020 CC BY 4.0 - in cooperation with Depts. of Cardiothoracic/ Vascular Surgery, General Surgery and Anesthesia, Örebro University Hospital and Örebro University, Sweden increases prehospital and in-hospital mortality. In a normal emergency department, with a few cases a day, this is not a problem. However, when you see 80-100 unstable patients from a massive explosion in a crowded city or neighborhood in a few hours, this can be overwhelming. And in these severely traumatized patients, traumatic bleeding remains a leading cause of potentially preventable death among severely traumatized patients [4]. Our problem in these patients is how to control anatomically and surgically the inaccessible bleeder, and usually these are intrathoracic, intra-abdominal, pelvic and zone I cervical bleeds. It is a surgical fact that in these situations control can be achieved effectively through an intraluminal approach using balloon catheters and catheter-guide wire techniques [5]. We use variable occlusion catheters to control intra-abdominal aortic, inferior vena cava injuries and subclavian artery injuries. Because of a lack of a proper angiography suite we carry out all these in the emergency department blindly. The aim is to have an endoluminal occlusion proximal to the suspected level of injury. Not having imaging guidance forces us to push the catheter to occlude the abdominal aorta as high as possible by mapping the catheter topographically to the abdominal surface before insertion, so that we know how far we need to push the catheter in different patients with different body sizes. This is not a perfect solution but it helps to buy time to resuscitate and transfer the patient to operative theatre.

With advances in imaging and in catheter-based endoluminal techniques such as Resuscitative Endovascular Balloon Occlusion of the Aorta (REBOA) [6], catheter-based embolization and hybrid operative theatres, it is now possible to provide better use of endovascular techniques in controlling and resuscitating inaccessible bleeders $[6,7]$. This can have a great impact, especially in managing mass causalities, by providing quick and precise control of bleeders and resuscitation. This helps the trauma team to have more time to operate on a large number of cases, so that patients are then better controlled and resuscitated. We believe that EVTM has an excellent place to operate in these mass causality situations. It is in these situations that the trauma team faces a large number of cases 


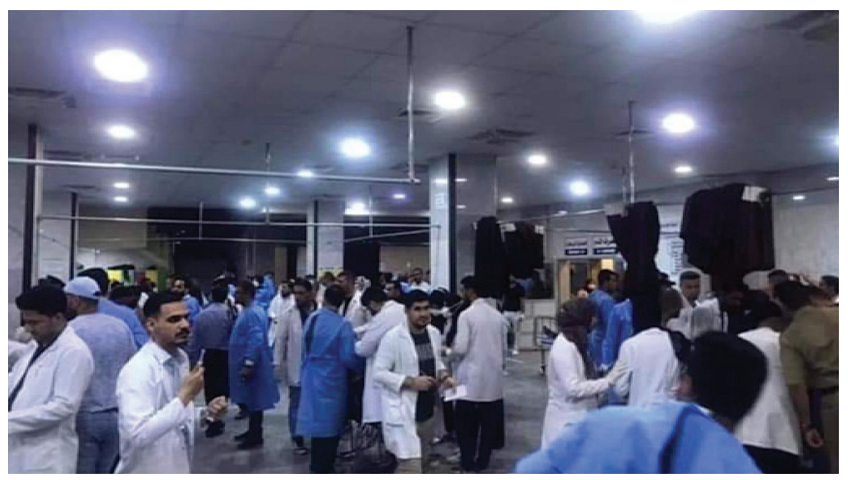

Figure 1 The medical city emergency department during receipt of mass casualties.

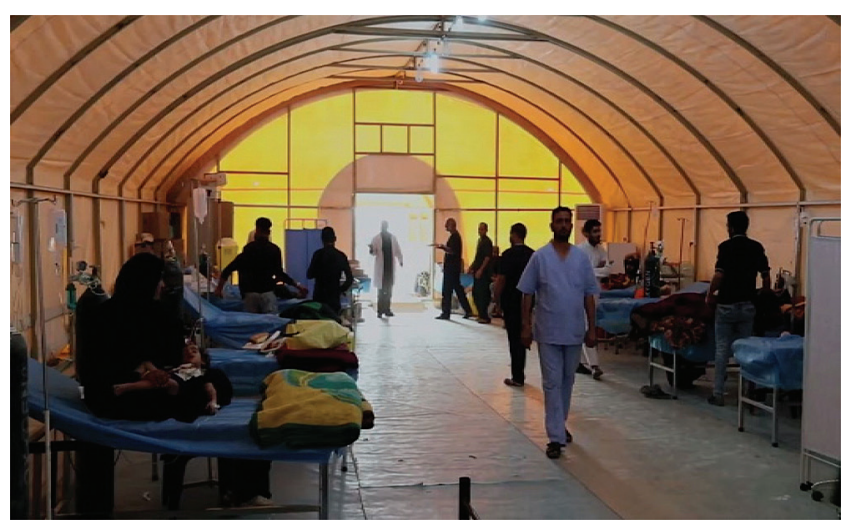

Figure 2 From a tent used as an emergency room in the battle field.

admitted over a short time, where most of them require bleeding control and resuscitation, and then need to be triaged to the surgery waiting queues. EVTM is useful in almost all situations as the techniques can be tailored to the available resources, similar to what we do in Iraq. They can be applied using a simple occlusion catheter, and they can be applied having all the modern resources of imaging endoluminal catheter-based intervention. This broad spectrum and the flexibility of the principles and tools of EVTM can help with the dissemination of practice guidelines in different places and scenarios.

\section{Ethics Statement}

(1) All the authors mentioned in the manuscript have agreed to authorship, read and approved the manuscript, and given consent for submission and subsequent publication of the manuscript.

(2) The authors declare that they have read and abided by the JEVTM statement of ethical standards including rules of informed consent and ethical committee approval as stated in the article.

\section{Conflicts of Interest}

The authors declare that they have no conflicts of interest.

\section{Funding}

The authors received no financial support for the research, authorship, and/or publication of this article.

\section{REFERENCES}

[1] DuBose J. "What's in a name?": a consensus proposal for a common nomenclature in the endovascular resuscitative management and REBOA literature. J Endovasc Resusc Trauma Manag 2017;1(1):9-12.

[2] Jacobs LM, Sinclair A, Beiser A, D’Agostino RB. Prehospital advanced life support: benefits in trauma. J Trauma. 1984;24(1):8-13.

[3] The ATLS Subcommittee ACoSCoT, group tIAw. Advanced trauma life support $\left(\right.$ ATLS $\left.^{\circledR}\right)$ : the ninth edition. J Trauma Acute Care Surg 2013;74(5):1363-6.

[4] Spahn DR, Bouillon B, Cerny V, et al. The European guideline on management of major bleeding and coagulopathy following trauma: fifth edition. Critical Care. 2019;23(1):98.

[5] Veith FJ, Sanchez LA, Ohki T. Technique for obtaining proximal intraluminal control when arteries are inaccessible or unclampable because of disease or calcification. J Vasc Surg. 1998;27(3):582-6.

[6] Horer T. Resuscitative endovascular balloon occlusion of the aorta (REBOA) and endovascular resuscitation and trauma management (EVTM): a paradigm shift regarding hemodynamic instability. Eur J Trauma Emerg Surg. 2018;44(4):487-9.

[7] Wijffels DJ, Verbeek DO, Ponsen KJ, Carel Goslings J, van Delden OM. Imaging and endovascular treatment of bleeding pelvic fractures: review article. Cardiovasc Intervent Radiol. 2019;42(1):10-8. 